\title{
Design of Reconfigurable Flight Controller Using Discrete Model Reference Adaptive Scheme
}

\author{
Seung-yong Hyung*, and Youdan Kim** \\ School of Mechanical and Aerospace Engineering \\ Seoul National University, Seoul, Korea
}

\begin{abstract}
In this paper, an adaptive control algorithm using system identification is proposed for an aircraft fault tolerant control system. A discrete state-space system is reformulated to be the ARX model which has the advantage in handing variable structure systems. Discrete model reference adaptive control is used to make the output of fault system follow the output of reference model. To validate the performance of the proposed control scheme, numerical simulations are performed for the high performance aircraft with control surface damage.
\end{abstract}

Key Word : ARX Model, Model Reference Adaptive Control

\section{Introduction}

Aircraft fault tolerant control system can be designed in two ways. The first approach is the hardware redundancy method; the second one is the analytical redundancy method[1]. Hardware redundancy methods deal with the faults by using the multiple sensors and actuators, and they are generally applied to the current aircraft redundant management systems[2]. However, the hardware redundancy method requires the additional cost and space due to the needs of multiple sensors/actuators. To overcome this shortcomings, the researches on the analytical redundancy methods, which diagnose the fault and redesign the controller using system mathematical model, have been widely studied.

In the early days, fault detection algorithms were based on the empirical database about the aircraft damage[3]. When the faults occur, the system detects the abnormal signals and isolates it by conditional classification. The compensation of the fault gets achieved by the pre-designed controller. This method is very simple and reliable. However in case the fault has never be experienced before nor is predictable, it is impossible to make alternative even if clear abnormalities are estimated on the system. Recently, lots of the flexible methods which does not meed the fault database are proposed to treat various faults, especially during the last decade, the reconfigurable control system with adaptive schemes has been studied. these approaches adaptive model following technique[4], eigenstructure assignment method[5]. variable structure control scheme[6] and neural network based adaptive control[7]. Those algorithms respond to the fault with intelligence in that it does not only detect the fault but generate appropriate control input compensating the fault effect.

In this study, discrete model reference adaptive control is used for the reconfiguration. Model reference adaptive control has the advantage that without the knowledge of the damaged system it can generate the proper input to follow the ideal model output. The structure of system is modified to ARX (Auto Regressove eXternal) model, which uses the input-output data as state variables. This modification strengthens the adaptability of the algorithm, when the system is changed its dimension by the damage, for example the actuator delay type fault. Numerical simulation is performed to validate the proposed reconfigurable controller. The control surface delay case is considered as the fault or damage of aircraft system.

* Currently Senior Engineer, Application Technology Lab, Samsung Electronics

** Professor, Seoul National University, corresponding author E-mail : ydkim@snu.ac.kr Tel : 02-880-7398 Fax : 02-887-2662 


\section{ARX Structure Using Input-Output Information}

In this section, the system model for system identification is derived using the input and output information. Consider the following discrete-time model of a system in the state-space form.

$$
\begin{aligned}
x(k+1) & =A x(k)+B u(k) \\
y(k) & =C x(k)
\end{aligned}
$$

where $x(k) \in R^{n}, u(k) \in R^{n}, y(k) \in R^{p}$ and $A, B, C$ are system matrix, input influence matrix, and output influence matrix, respectively.

Since the discrete system matrix $A$ is a state transition matrix, it is always nonsingular. Using Eqs. (1) and (2), the output variables can be described using the state variables and inputs as follows:

$$
y(k-j)=C A^{-j} x(k)-\sum_{i=0}^{j-1} C A^{-j+1} B u(k-i-1)
$$

The above equation can be rewritten as follows

where

$$
Y(k)=H x(k)+P U(k)
$$

$$
\begin{gathered}
Y(k)=\left[\begin{array}{c}
y(k) \\
y(k-1) \\
\vdots \\
y(k-q+1)
\end{array}\right], H\left[\begin{array}{c}
C \\
C A^{-1} \\
\vdots \\
C A^{-q+1}
\end{array}\right], U(k)=\left[\begin{array}{c}
u(k-1) \\
u(k-2) \\
\vdots \\
u(k-q+1)
\end{array}\right] \\
P=\left[\begin{array}{cccc}
0 & 0 & 0 & 0 \\
-C A^{-1} B & 0 & 0 & 0 \\
\vdots & & \ddots & \vdots \\
-C A^{-q+1} B-C A^{-q+2} B \cdots & C A^{-1} B
\end{array}\right]
\end{gathered}
$$

Note from Eq. (4) that the state variable $x(k)$ can be obtained from the given input and output data. If the size of matrix $\mathrm{H}$ is $n \times n$ and the rank of matrix $A$ is $n$, then $x(k)$ can be uniquely determined by using $Y(k)$ and $U(k)$. Using Eq. (1) and Eq. (4), the following equations can be obtained.

$$
\begin{aligned}
& x(k)=H^{\dagger}(Y(k)-P U(k)) \\
& x(k+1)=A H^{\dagger}(Y(k)-P U(k))+B u(k) \\
& y(k+1)=C A H^{\dagger}(Y(k)-P U(k))+C B u(k)
\end{aligned}
$$

where $H^{\dagger}$ is the pseudo-inverse of matrix $H$.

Using the definition of $U(k)$, the following equation can be obtained.

$$
\left[\begin{array}{c}
Y(k+1) \\
U(k+1)
\end{array}\right]=\left[\begin{array}{c}
J_{p}+I_{p} C A H^{\dagger}-I_{p} C A H^{\dagger} P \\
0 \\
J_{m}
\end{array}\right]\left[\begin{array}{l}
Y(k) \\
U(k)
\end{array}\right]+\left[\begin{array}{c}
I_{p} C B \\
I_{m}
\end{array}\right] u_{k}
$$

where

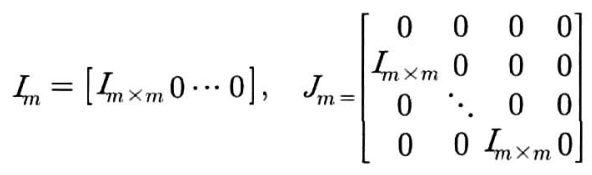

and $I_{m \times m}$ is an $m \times m$ identity matrix. Equation (8) will be used for reconfigurable control. 


\section{Discrete Model Reference Adaptive Control}

Multi-input multi-output discrete linear system plant can be described by Eq.(8) and it can be rewritten as follows[8].

$$
\begin{aligned}
& x_{s}(k+1)=A_{s} x_{s}(k)+B_{s} u_{s}(k) \\
& y_{s}(k+1)=C_{s} x_{s}(k)+D_{s} u_{s}(k)
\end{aligned}
$$

where

$$
x_{s}=\left[\begin{array}{c}
Y(s) \\
U(s)
\end{array}\right], A_{s}=\left[\begin{array}{cc}
J_{p}+I_{p} C A H^{\dagger}-I_{p} C A H^{\dagger} P \\
0 & J_{m}
\end{array}\right], B_{s}=\left[\begin{array}{c}
I_{p} C B \\
I_{m}
\end{array}\right], C_{s}=I, D_{s}=0
$$

If there is sudden fault on the system, the parameters of this matrix system become unknown.

Consider the reference model for the system to follow. An asymptotically stable reference model is represented by the following equation.

$$
\begin{aligned}
& x_{m}(k+1)=A_{m} x_{m}(k)+B_{m} r_{m}(k) \\
& y(k+1)=C_{m} x_{m}(k)+D_{m} r_{m}(k)
\end{aligned}
$$

The objective of the control considered in this study is to determine adequate control input to make the output $y_{s}$ follow the model output. The output error is defined as

$$
e(k)=y(k)-y_{m}(k)
$$

It is noticeable that $y_{m}$ is not just system output. vector $y_{m}$ is structured the input-output sequence vector from the ideal model.

In this study, we adopt the adaptive controller composed of a gain $K$ and compensator parameters $K_{u}, K_{y}$ described as

$$
\begin{gathered}
Z(k+1)=\left[\begin{array}{l}
\zeta_{1}(k+1) \\
\zeta_{2}(k+1)
\end{array}\right]=\left[\begin{array}{l}
F 0 \\
0
\end{array}\right]\left[\begin{array}{l}
\zeta_{1}(k) \\
\zeta_{2}(k)
\end{array}\right]+G\left[\begin{array}{l}
u(k) \\
y(k)
\end{array}\right] \\
\Omega(k)=\left[\begin{array}{c}
\omega_{1}(k) \\
\omega_{2}(k)
\end{array}\right]=\left[\begin{array}{cc}
K_{u} & 0 \\
0 \\
K_{y}\left[\begin{array}{l}
\zeta_{1}(k) \\
\zeta_{2}(k)
\end{array}\right]
\end{array}\right]
\end{gathered}
$$

where $F$ is stable matrix, and $(F, G)$ is controllable. Supplementary Compensator is needed for discrete adaptive stability of model following controller. Then, parameters $\Theta$ of the controller can be defined as

$$
\Theta(k)=\left[K(k) K_{u}(k) K_{y}(k)\right]
$$

Let us define the vector $\Phi(k)$ as

$$
\Phi(k)=\left[r(k)^{T} \zeta_{1}(k)^{T} \zeta_{2}(k)^{T}\right]
$$

Then, the feedback input may be represented as

$$
\begin{aligned}
u(k) & =K(k) r(k)+K_{u}(k) \zeta_{1}(k)+K_{y}(k) \zeta_{2}(k)-\alpha \gamma e(k) \Phi^{T} \Phi \\
& =\Theta(k) \Phi(k)-\alpha \gamma e(k) \Phi^{T^{T} \Phi}
\end{aligned}
$$


The stability of the closed-loop system is guaranteed if $\alpha>1 / 2$ and $\gamma>0$. The detailed proof is summarized in Appendix. Therefore, by using the above control law the output of the system can follow the model output even when fault occurred. Note that, the parameter can be updated using the following adaptive rule.

$$
\Theta(k+1)=\Theta(k)-\gamma e(k) \Phi^{T}
$$

The structure of the adaptive control system is shown in Fig. 1.

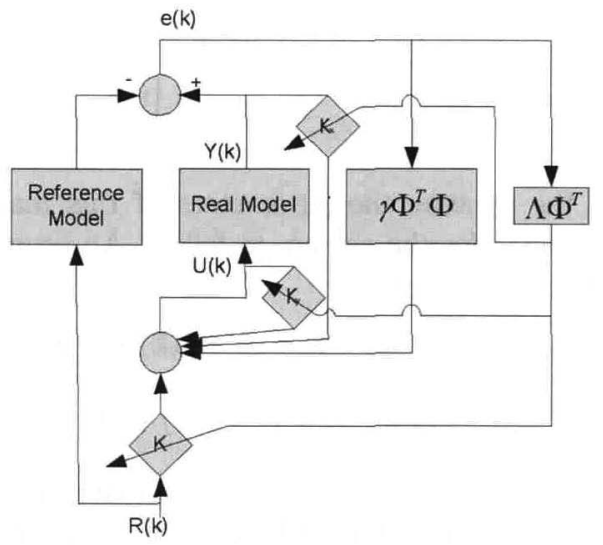

Fig. 1. Discrete Model Reference Adaptive Control

\section{Numerical Simulation}

\section{A. Reconfigurable Controller}

Numerical simulation is performed to verify the performance of the proposed adaptive reconfigurable flight controller. For numerical simulation, high performance aircraft $\mathrm{F}-16$ with control surface damage was considered. The flight condition was a level flight at the velocity of $502 \mathrm{ft} / \mathrm{sec}$, sea level, and cg at $0.35 \bar{c}$. The elevator damage of $60 \%$ occurred at 4 seconds. The following pitch rate command was considered.

$$
q=\left\{\begin{array}{l}
10^{\circ} / s 0 \leqq t<5 \\
-10^{\circ} 5 \leqq t<15
\end{array}\right.
$$

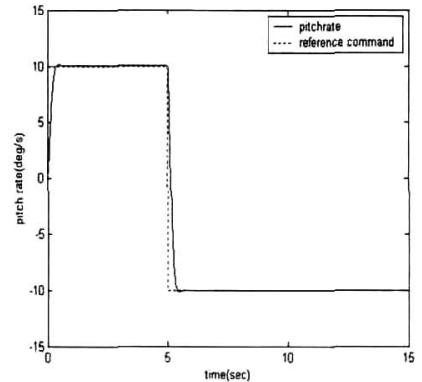

(a)Normal case

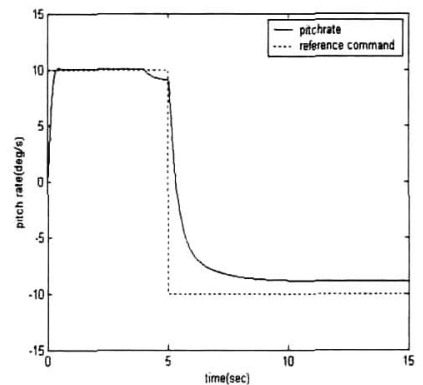

(b)Faulty case

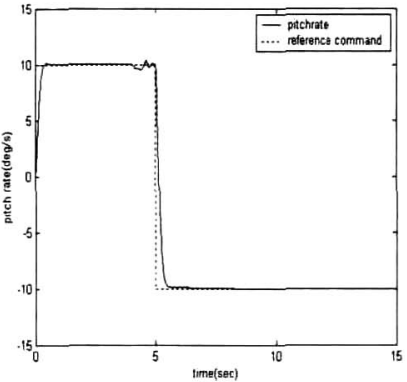

(c)Reconfiguration

Fig. 2. Pitch Rate Responses 


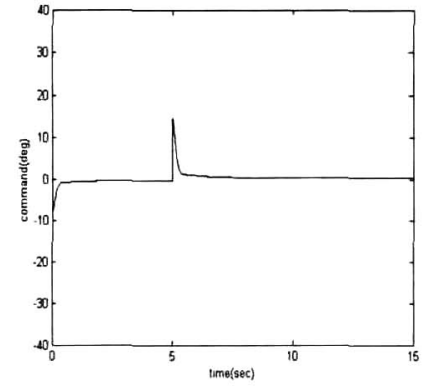

(a)Normal case

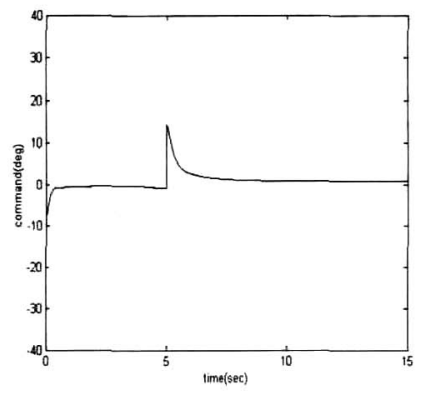

(b)Faulty case

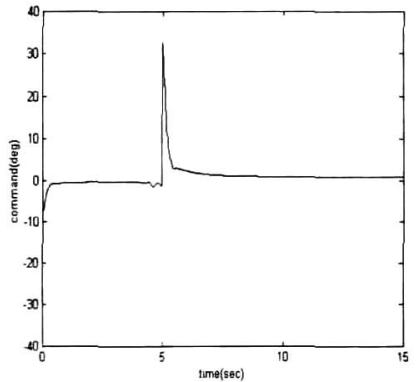

(c)Reconfiguration

Fig. 3. Elevator Command

Figure 2(a) shows the pitch rate response of the normal system using the LQ tracking controller to follow the reference command. Control augmentation system makes the aircraft follow the command pitch rate. Figure 2(b)shows the control surface damage case. After the damage of the actuator(at $4 \mathrm{sec})$, the pitch rate of the system slows down to $9 \mathrm{deg} / \mathrm{s}$, and the system cannot follow the reference input because of the lack of the feed-forward control input due to damage. To overcome this problem, the controller must be redesigned to identify the control surface efficiency reduction. More control input should be applied to compensate the effects of the damage. Figure 2(c) shows the reconfigurable control system case. At the instant of fault occurrence, the reconfigurable system make the system follow the trajectory of reference input. Figure 2(c) shows that the proposed reconfigurable controller exhibits the good tracking performance. Figure 3 shows the control input histories.

\section{B. Comparison between the conventional MRAC and modified MRAC}

The advantage of the proposed discrete MRAC method is that it is not required to assign the system dimension. The ARX model is expressed by the past input-output data and the dimension of system model is determined by the data size of the gathering input output information. It is natural that the data size q must be larger than the system dimension, and the accurate size of dimension does not need to be known. Generally, fault changes the system parameter values as well as the dimension of the system. For example, the delay type fault requires one more state variable. However, the conventional MRAC cannot manage such type of faults because the exact information of dimension is not acquired. Modified ARX structure proposed in this study can deal with this case.

To compare the performance between the conventional MRAC and the modified MRAC, the longitudinal model of the high performance aircraft with fall-off of the actuator speed was considered.

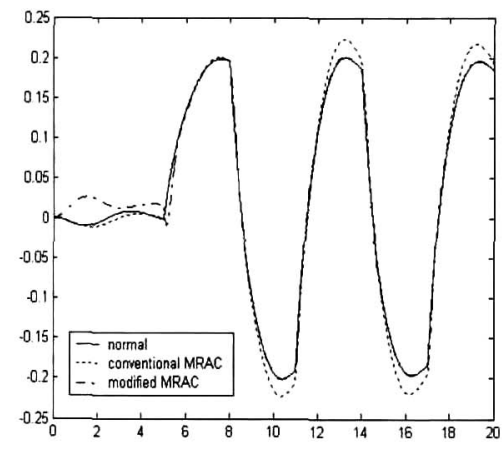

Fig. 4. Pitch Angle Response with Reconfiguration

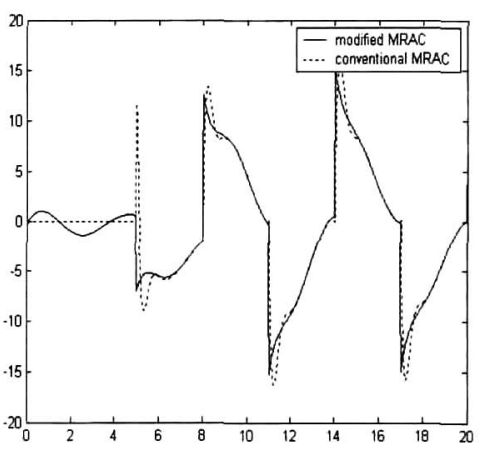

Fig. 5. Input Response with Reconfiguration 
The flight condition was a level flight at the velocity of $502 \mathrm{ft} / \mathrm{sec}$, sea level, and $\mathrm{cg}$ at $0.35 \bar{c}$. We consider the elevator damage case of the first order type at the initial time. The dimension of the original system model is $4 \times 4$. However, the dimension of the model after the fault becomes $5 \times 5$. It is known that the conventional MRAC algorithm has good tracking performance when the system is controllable, observable and system dimension is not varied. Figure 4 shows that conventional MRAC has difficulty in finding the good control input because the system dimension is changed, Modified MRAC follows the reference model accurately. Note that the modified method requires more parameters than the conventional method. And therefore, the convergence time of the modified method is a little bit longer than that of the conventional MRAC. Figure 5 shows the input response with reconfiguration.

\section{Conclusions}

An adaptive reconfigurable control system based on system identification is proposed for the aircraft fault tolerant control system. The derived system model has the same structure as an ARX model that any conventional recursive system identification technique can be applied. To construct the stable tracking system, a discrete model reference adaptive control method is combined to this structure. The performance of the proposed fault tolerant control scheme is verified using a high performance aircraft with actuator damage.

\section{Acknowledgement}

This research was performed for Smart UAV Development Program, one of the 21 Century Frontier R\&D Programs funded by the Ministry of Commerce, Industry and Energy of Korea.

\section{References}

1. R. N. Clark, P. M. Frank, and R. J. Patton, Fault Diagnosis in Dynamic Systems: Theory and Application, Prentice Hall, New York, 1996, Chap. 1.

2. R. C. Hammett, "Ultra-Reliable Real-Time Control Systems-Future Trends", IEEE Aerospace and Electronic Systems Magazine, Vol. 14, No. 8, 1989, pp. 31-36.

3. R. J. Patton, and J. Chen, "Robust Fault Detection of Jet Engine Sensor Systems Using Eigenstructure Assignment", Journal of Guidance, Control and Dynamics, Vol. 15, No. 6, 1991, pp. 1491-1497.

4. W. D. Morse, and K. A. Ossman, "Model Following Reconfigurable Flight Control System for the AFTI/F-16", Journal of Guidance, Control, and Dynamics, Vol. 13, No. 6, 1999, pp. 969-976.

5. J. Jiang, "Design of Reconfigurable Control System Using Eigenstructure Assignment", International Journal of Control, Vol 59, No. 2 , 1994, pp. 395-410.

6. D. Kim, and Y. Kim, "Robust Variable Structure Controller Design for Fault Tolerant Flight Control", Journal of Guidance, Control, and Dynamics, Vol. 23, No. 3, 2000, pp. 430-437.

7. D. H. Shin, and Y. Kim, "Reconfigurable Flight Control System Design Using Adaptive Neural Networks", IEEE Transactions on Control Systems Technology, Vol. 12, No. 1, 2004, pp. 87-100.

8. S. Hiroshi, S. Yu, F. Toru, and M. Genmon, "Discrete-time Algorithm for Generalized Simplified Adaptive Control and Its Application to DC Motor Control", IEEE IECON 22nd International Conference, august 1996, pp. 332-339.

9. B. L. Stevens and F. L. Lewis, Aircraft Control and Simulation, John wiley \& Sons, Inc., New York, 1992, Chaps. 4 and 5.

10. C. G. Jeong, Stable Model Reference Adaptive Control of Linear Discrete Timeinvariant Multivariable System, MS Thesis, Department of Electrical Engineering, Seoul National University, Korea, 1984.

11. R. B. Robert, "Explicit Solution of the Discrete-Time Lyapunov Matrix Equation and Kalman-Yakubovich Equations", IEEE Transactions on Automatic Control, Vol. 26, No. 6, 1981, pp. $1291-1294$. 


\section{Appendix A. Stability of Discrete Adaptive Control[10]}

Consider the reference model(A1) and the real system on which reconfigurable algorithm is $\operatorname{applied(A2)~.~}$

$$
\begin{gathered}
x_{t o t m}(k+1)=A_{t o t} x_{t o t m}(k)+B_{t o t} K^{*} r(k) \\
x_{t o t}(k+1)=A_{t o t} x_{t o t}(k)+B_{t o t}\left(K^{*} r(k)+\tilde{\Theta}(k) \Phi(k)-\alpha \gamma e(k) \Phi(k)^{T} \Phi(k)\right)
\end{gathered}
$$

where

$$
\begin{gathered}
A_{t o t}=\left[\begin{array}{ccc}
A_{s} & B_{s} K_{u}^{*} & B_{s} K_{y}^{*} \\
0 & F+G K_{u}^{*} & G K_{y}^{*} \\
G C_{s} & G D_{s} K_{u}^{*} & F+G D_{s} K_{y}^{*}
\end{array}\right], \quad B_{t o t}=\left[\begin{array}{c}
B_{s} \\
G \\
G D_{s}
\end{array}\right], \quad x_{t o t}=\left[\begin{array}{c}
x_{s}(k) \\
\zeta_{1}(k) \\
\zeta_{2}(k)
\end{array}\right] \\
E_{t o t}=x_{t o t}(k)-x_{t o t m}(k) \\
C_{t o t}=\left[\begin{array}{ll}
I_{m \times m} & 0
\end{array}\right] \\
S(k)=\tilde{\Theta}(k) \Phi(k)-\alpha \gamma e(k) \Phi^{T} \Phi \\
\Theta^{*}=\left[\begin{array}{ll}
K^{*} K_{u}^{*} K_{y}^{*}
\end{array}\right] \\
\tilde{\Theta}(k)=\Theta(k)-\Theta^{*}
\end{gathered}
$$

Note that Matrices $K^{*}, K_{u}^{*}, K_{y}^{*}$ are the parameters to be identified. And adaptive law is proposed as follows.

$$
\Theta(k+1)=\Theta(k)-\gamma e(k) \Phi^{T}
$$

The error equation between the model and the system can be expressed as follows.

$$
\begin{gathered}
E(k+1)=A_{t o t} E(k)+B_{t o t}\left(\tilde{\Theta}(k) \Phi(k)-\alpha \gamma e(k) \Phi^{T} \Phi\right) \\
e(k)=C_{t o t} E(k)+D_{t o t}\left(\tilde{\Theta}(k) \Phi(k)-\alpha \gamma e(k) \Phi^{T} \Phi\right.
\end{gathered}
$$

To prove the stability of the closed-loop system, consider the following tentative Lyapunov function is selected.

$$
V(E(k), \tilde{\Theta}(k))=E^{T}(k) P E(k)+\operatorname{Tr}\left[\tilde{\Theta}(k) \gamma^{-1} \tilde{\Theta}(k)\right]
$$

By Kalman-Szego-popov lemma, there exist symmetric positive matrices $P, K$, and $L$ such that

$$
\begin{aligned}
& A_{t o t}^{T} P A_{t o t}-P=-L L^{T} \\
& A_{t o t}^{T} P B_{t o t}=C_{t o t}^{T}+L K \\
& B_{t o t}^{T} P B_{t o t}=D_{t o t}+D_{t o t}^{T}-K^{T} K
\end{aligned}
$$

where $A_{t o t}, B_{t o t}$ is controllable and stable[11].

To verify the stability of the system, $\Delta V(k)<0$ must be shown.

$$
\begin{aligned}
\Delta V(k)= & V(k+1)-V(k) \\
= & E^{T}(k+1) P E(k+1)+\operatorname{Tr}\left[\widetilde{\Theta^{T}}(k+1) \gamma^{-1} \tilde{\Theta}(k+1)\right] \\
& -E^{T}(k) P E(k)-\operatorname{Tr}\left[\tilde{\Theta}(k) \gamma^{-1} \tilde{\Theta}(k)\right] \\
= & \left(A_{t o t} E(k)+B_{t o t} S(k)\right)^{T} P\left(A_{t o t} E(k)+B_{t o t} S(k)\right)-E^{T}(k) P E(k) \\
& +\operatorname{Tr}\left[(\tilde{\Theta}(k)+\Delta \tilde{\Theta}(k))^{T} \gamma^{-1}(\tilde{\Theta}(k)+\Delta \tilde{\Theta}(k))\right]-\operatorname{Tr}\left[\tilde{\Theta}(k) \gamma^{-1} \tilde{\Theta}(k)\right] \\
= & E^{T}(k)\left[A_{t o t}^{T} P A_{t o t}-P\right] E(k)+2 E^{T}(k) A_{t o t}^{T} P B_{t o t} S(k) \\
& +S^{T(k)} B_{t o t}^{T} P B_{t o t} S(k)+\operatorname{Tr}\left[\Delta \widetilde{\Theta}^{T}(k) \gamma^{-1} \Delta \tilde{\Theta}(k)\right] \\
& +\operatorname{Tr}\left[\Delta \widetilde{\Theta}^{T}(k) \gamma^{-1} \tilde{\Theta}(k)\right]+\operatorname{Tr}\left[\widetilde{\Theta}^{T}(k) \gamma^{-1} \Delta \tilde{\Theta}(k)\right]
\end{aligned}
$$


Substituting Eq.(A6) into Eq.(A7) yields

$$
\begin{aligned}
\Delta V(k)= & -\left[E^{T}(k) L-S^{T}(k) K^{T}\right]\left[E^{T}(k) L-S^{T}(k) K^{T}\right]^{T}+2 E^{T}(k) C_{t o t}^{T} S(k) \\
& +S^{T}(k)\left(D_{t o t}+D_{t o t}^{T}\right) S(k)+\operatorname{Tr}\left[\Delta \widetilde{\Theta}^{T}(k) \gamma^{-1} \Delta \tilde{\Theta}(k)\right] \\
& +\operatorname{Tr}\left[\Delta \widetilde{\Theta}(k) \gamma^{-1} \widetilde{\Theta}(k)\right]+\operatorname{Tr}\left[\widetilde{\Theta}^{T}(k) \gamma^{-1} \Delta \widetilde{\Theta}(k)\right]
\end{aligned}
$$

From Eq.(A2) the second term of Eq.(A8) can be expressed as follows

$$
\begin{aligned}
& 2 E^{T}(k) C_{t o t}^{T} S(k)+S^{T}(k)\left(D_{t o t}+D_{t o t}^{T}\right) S(k) \\
= & \left(2 E^{T}(k) C_{t o t}^{T}+S^{T}(k)\left(D_{t o t}+D_{t o t}^{T}\right)\right) S(k) \\
= & 2 e^{T}(k) S(k)
\end{aligned}
$$

Using adaptive rule described in Eq.(A3) the third term of Eq.(A8) can be extended as follows

$$
\begin{aligned}
& \operatorname{Tr}\left[\Delta \widetilde{\Theta^{T}}(k) \gamma^{-1} \Delta \tilde{\Theta}(k)\right]+\operatorname{Tr}\left[\Delta \widetilde{\Theta^{T}}(k) \gamma^{-1} \tilde{\Theta}(k)\right]+\operatorname{Tr}\left[\widetilde{\Theta^{T}}(k) \gamma^{-1} \Delta \tilde{\Theta}(k)\right] \\
& =\operatorname{Tr}\left[\Phi(k) e^{T}(k) \gamma e(k) \Phi(k)^{T}-\Phi(k) e^{T}(k) \tilde{\Theta}(k)-\widetilde{\Theta}^{T}(k) e(k) \Phi^{T}(k)\right]
\end{aligned}
$$

where

$$
\begin{aligned}
& 2 e^{T}(k) S(k) \\
& +\operatorname{Tr}\left[\Phi(k) e^{T}(k) \gamma e(k) \Phi^{T}(k)-\Phi(k) e^{T}(k) \tilde{\Theta}(k)-\widetilde{\Theta}^{T}(k) e(k) \Phi^{T}(k)\right] \\
= & 2 e^{T}(k)\left(\tilde{\Theta}(k) \Phi(k)-\alpha \gamma e(k) \Phi^{T} \Phi\right)+\operatorname{Tr}\left[\Phi(k) e^{T}(k) \gamma e(k) \Phi^{T}(k)\right. \\
& \left.-\Phi(k) e^{T}(k) \widetilde{\Theta}^{T}(k)-\widetilde{\Theta}^{T}(k) e^{T}(k) \Phi^{T}(k)\right] \\
= & -2 \alpha e^{T}(k) \gamma e(k) \Phi^{T}(k) \Phi(k)+\operatorname{Tr}\left[\Phi(k) e^{T}(k) \gamma e(k) \Phi^{T}(k)\right] \\
& +2 e^{T}(k) \tilde{\Theta}(k) \Phi(k)+\operatorname{Tr}\left[-\Phi(k) e^{T}(k) \tilde{\Theta}(k)-\widetilde{\Theta}^{T}(k) e(k) \Phi^{T}(k)\right] \\
= & (-2 \alpha+1) e^{T}(k) \gamma e(k) \Phi^{T}(k) \Phi(k)
\end{aligned}
$$

Substitution of (A8) yields

$$
\Delta V(k)=-\left[E^{T}(k) L-S^{T}(k) K^{T}\right]\left[E^{T}(k) L-S^{T}(k) K^{T}\right]^{T}+(-2 \alpha+1) e^{T} \gamma e \Phi^{T} \Phi \leqq 0
$$

If $\alpha>1 / 2$, then $V(k) \rightarrow 0$ as $k \rightarrow \infty$. 